\title{
ON THE ROTATION MODEL IN VLBI GEOPHYSICAL ANALYSIS
}

\author{
Y. F. Xia \\ Astronomy Department \\ Nanjing University \\ People's Republic of China
}

The basic observables of VLBI for geophysics are the delay and the delay rate. These two basic observables depend on relative geometric relations between the baseline vector and the source vector. The transformation of baseline orientation from the terrestrial coordinate system, in which the stations are located, to the celestial coordinate system, in which VLBI observables are calculated, involves a number of models. One of them is the rotation model.

In this paper, we first describe the relations among various reference poles for the deformable earth and the liquid core earth; then the rotation model in VLBI geophysical analysis is discussed. It points out:

1. If the nutation given is the nutation of the angular momentum pole, and if the diurnal spin matrix rotates about the pole, then the rotation model can be represented as

$$
\mathbf{B}_{s f}=P N S D W \mathbf{B}_{b f},
$$

where $\mathbf{B}_{s f}$ and $\mathbf{B}_{b f}$ represent the baseline vector given in the space-fixed and body-fixed coordinate system, respectively, and $D$ is the diurnal polar motion matrix. It is defined as the transformation in the body-fixed coordinate system from the angual momentum pole to the angular momentum pole with force-free polar motion. Its amplitude is that of the forced polar motion of the angular momentum pole.

2. If the diurnal spin matrix rotates about the angular momentum pole with force-free polar motion, then the rotation model can be represented as

$$
\mathbf{B}_{s f}=P N O_{p} S^{\prime} W \mathbf{B}_{b f},
$$

where $O_{p}$ is called the Oppolzer term transformation matrix. Its amplitude is that of the nutation Oppolzer term of Celestial Ephemeris Pole (CEP). $O_{p}$ can be predicted.

3. If the force nutation of the mean figure pole is given, then the rotation model can be represented as

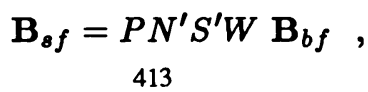


which include neither the diurnal polar motion matrix nor the Oppolzer term transformation matrix.

4. For the liquid core earth, the matrix that transforms the angular momentum pole with force-free polar motion into a Chandler angular momentum pole is neglected. Its amplitude is that of the nearly diurnal free polar motion of the angular momentum pole in the body-fixed coordinate system and is that of the free core nutation of the mean figure pole in the space-fixed coordinate system.

The research of liquid core earth will provide more perfect rotation model for VLBI geophysical analysis.

\section{ACKNOWLEDGMENT}

The author wishes to acknowledge the help of Professor W. Cannon in discussions of reference system. 